\title{
AMNISTIIA INTERNACIONAL Y EDUCACIÓN EN DERECHOS HUMANOS
}

\section{Iñaki Gómez}

\author{
Equipo de Educación de Amnistia Internacional
}

RESUMEN. Amnistía Internacional es un movimiento mundial de activistas voluntarios que se esfuerzan por prevenir algunas de las peores violaciones de derechos humanos que cometen ciertos gobiernos, trabajando principalmente por la obtención de la libertad de todos los presos de conciencia, logrando juicios imparciales para los presos políticos, luchando por la abolición de la pena de muerte y la erradicación de la tortura, así como persigue también terminar con las ejecuciones extrajudiciales y las "desapariciones". Para alcanzar estos fines, Amnistía Internacional se esfuerza por promover todos los derechos humanos consagrados en la Declaración Universal de Derechos Humanos y otras normas internacionales mediante programas de educación, no sólo en el ámbito escolar, propiciando la sensibilización y formación de la sociedad, buscando integrar la educación y la acción, por lo que posibilita siempre la participación activa de sus colaboradores en la defensa de los derechos humanos. El presente artículo desarrolla estos puntos de partida sobre la importancia de la educación en la lucha por la defensa de los derechos humanos, desarrollando sus objetivos prioritarios, las dinámicas de trabajo habituales así como algunas sugerencias de material didáctico útil desde el punto de vista de la organización, es decir, desde la confianza en el valor preventivo de la educación.

ABSTRACT. Amnesty International is a worldwide movement of volunteer activists, who make efforts to prevent some of the worst human rights violations committed by some governments, working mainly to obtain freedom for all the prisoners of conscience, getting impartial trials for political prisoners, fighting for the death penalty abolition and the eradication of torture. A.I. looks for stopping the extrajudicial executions and "disappearance". In order to get these proposals, A.I. makes efforts to promote every human rights consecrated in the Universal Declaration of Human Rights and another international rules, using educative programs, not only in the school, seeking for the education of the society, looking for joining education with action, always allowing the active participation in the defence of human rights to its helpers. This article explains these principles on the importance of the education in the fight for the human rights, developing its main objectives, usual work and some suggestions about useful didactic material from the point of view of A.I, that is to say, the confidence in the preventive worth of education. 
El objetivo de Amnistía Internacional (Al) es contribuir a que se observen en todo el mundo los derechos que establece la Declaración Universal de Derechos Humanos. De conformidad con este objetivo, el estatuto de la Organización establece una doble tarea: promover el conocimiento de la Declaración Universal de Derechos Humanos y la adhesión a las normas emanadas de dichos derechos, así como oponerse activamente a la violación de determinados derechos civiles y políticos.

Desde una organización de defensa de los Derechos Humanos como Al, fundada en 1.961 y con más de 1.100 .000 socios en todo el mundo, creemos firmemente que es totalmente necesario trabajar para detener las violaciones de derechos humanos que ya se producen (en su conocida labor en favor de los presos de conciencia, los presos políticos y contra la tortura y las ejecuciones). En consecuencia, también es importante trabajar de forma preventiva y permanente con la finalidad de evitar que estas violaciones se produzcan en el futuro.

Consideramos, entonces, que la educación en derechos humanos es una buena herramienta para la prevención de las violaciones de derechos humanos, fomentando la conducta y las actitudes basadas en la tolerancia, la solidaridad y el respeto.

La educación en derechos humanos, tal y como la entendemos desde $\mathrm{Al}$, pretende informar de estos derechos a las personas de todas las edades y profesiones, y muy especialmente a los jóvenes, para que puedan valorar y defender sus derechos y los de los demás, fomentando con ello:

- la comprensión de los conceptos básicos que subyacen tras los derechos humanos como son la solidaridad, la igualdad y la tolerancia;

- la comprensión de la relación entre derechos individuales, de grupos y nacionales;

- el conocimiento de los principales acontecimientos históricos en el desarrollo de los derechos humanos y la comprensión de la gama de declaraciones, convenciones y pactos sobre estos derechos;

- el conocimiento de las principales infracciones de los derechos humanos;

- y la preocupación activa y solidaria por quienes sufren las violaciones de sus derechos.

De este modo, Amnistía Internacional, con el trabajo de sus equipos de formación y educación y el esfuerzo de difusión pública de su activismo, pretende crear una cultura preventiva en materia de derechos humanos, algo fundamental para erradicar la violación de los mismos. Con ella conseguiremos conocer todos los derechos humanos, distinguirlos, actuar en su favor $y$, sobre todo, concienciarnos del inmenso privilegio que es poder disfrutarlos en un mundo sometido a tantas injusticias como el nuestro.

\section{Planteamientos de partida: educación, formación y sensibilización}

El mandato de Al, esencia del trabajo y fines de la organización, tiene por objetivo contribuir al respeto en todo el mundo de los derechos proclamados en la Declaración Universal de Derechos Humanos, como ya hemos señalado anteriormente. De conformidad con este propósito, Al intenta fomentar el conocimiento, la comprensión y la adhesión a las normas de derechos humanos en general y actúa concretamente 
para oponerse a las violaciones de ciertos derechos civiles y políticos. En consecuencia, las actividades centrales de AI hasta la década de los ochenta estaban dirigidas principalmente a denunciar y oponerse a las violaciones de algunos derechos civiles y políticos que se cometían en el mundo. Durante el último decenio se ha ido comprendiendo, de modo creciente, que la lucha mundial contra las violaciones de derechos humanos puede cobrar renovado impulso si se la combina con un tratamiento preventivo más vigoroso, como la educación en derechos humanos (EDH) o la sensibilización sobre los derechos humanos.

En consecuencia, Al entiende la educación o formación en derechos humanos como un programa amplio que debe proporcionar los medios necesarios para adquirir conocimiento y comprensión de los derechos humanos y que debe contribuir a fomentar los valores y actitudes que conducen al respeto de esos derechos. Para tener buenos resultados, este programa debe mantenerse durante cierto periodo de tiempo, repetirse periódicamente e incluir la interacción entre dos partes: la que imparte la formación y la que la recibe; La EDH incluye también el desarrollo de aptitudes básicas como el pensamiento crítico, la comunicatividad y la habilidad para negociar y para resolver problemas, todos ellos esenciales para un activismo competente y para la participación en el proceso de toma de decisiones.

Para ello parte, primeramente, de la formación y educación de sus socios y miembros activos de los diferentes grupos. El estudio y reflexión de diferente documentación sobre la situación de los derechos humanos en el mundo, talleres de trabajo, análisis de material audiovisual, etc, permiten a los activistas de la organización mejorar su conocimiento al respecto y difundirlos adecuadamente mediante charlas abiertas a diferentes colectivos, manteniendo su presencia en todos aquellos foros que son propicios para difundir una cultura positiva de respeto a los derechos humanos. En todas esas ocasiones, se facilita material diverso sobre diferentes campañas de la organización y se posibilita el compromiso inmediato de aquellas personas que lo deseeen, mediante la recogida de firmas o el envío de cartas y telegramas a autoridades, siempre dentro de la resolución pacífica de algún caso. Propiciar el paso de la educación a la acción es una premisa de trabajo importante.

En esa misma línea, Al persigue la sensibilización sobre los derechos humanos entendida como la interiorización a medio plazo de la información recibida sobre estos derechos. El trabajo de sensibilización sobre los derechos humanos incluye, por ejemplo, una exposición de carteles, un programa de radio o una charla como las ya citadas. No se requiere, en principio, la participación activa del público y el acto puede ser único. Esto significa que los actos individuales relacionados con la sensibilización sobre los derechos humanos no tendrán, normalmente, una influencia a largo plazo sobre las actitudes ni la conducta de los individuos, pero, como es propio de la acción educativa, los frutos pueden no ser inmediatos, pero queda un trabajo importante realizado que, con mucha frecuencia, tras un periodo de reflexión, se traduce en nuevos apoyos.

En consecuencia, se produce una superposición natural entre la educación en derechos humanos y la sensibilización sobre los derechos humanos. En algunos países será preciso comenzar con trabajo de sensibilización sobre los derechos humanos a fin de crear el ámbito deseado para poder desarrollar y poner en práctica un programa sostenible de educación en derechos humanos. 


\section{Una mirada de cerca a la educación en Derechos Humanos}

La EDH consiste entonces en ayudar a comprender la importancia de los derechos humanos y proporcionar los medios necesarios para adquirir los conocimientos, actitudes y habilidades necesarios para promover y proteger estos derechos.

Además de la formación interna de sus socios y de las actividades de difusión ya citadas, otro ejemplo práctico de $\mathrm{EDH}$ desde $\mathrm{Al}$ es la realización de cursos periódicos de formación para policías sobre cómo respetar los derechos de los detenidos o sobre la obligación ineludible de denunciar a colegas responsables de malos tratos. Este aspecto de la EDH se cuida cada vez más, conscientes de lo eficaz y necesario de su puesta en práctica.

Y por otra parte, se considera de gran importancia el desarrollo de un programa transversal de estudios y la elaboración de materiales de enseñanza para niños y jóvenes acerca de la Declaración Universal de Derechos Humanos, aspecto este que desarrollaremos más adelante. También forman parte del trabajo de los educadores en derechos humanos las gestiones ante las autoridades pertinentes para lograr que incorporen el tema de los derechos humanos a un programa específico de formación o educación, tanto para adultos como para jóvenes, en todos aquellos currículos en los que resulta oportuno.

Destacar también que si bien la EDH no entraña, en principio, trabajo sobre casos individuales de violaciones de derechos, sí se puede, junto con otras labores preventivas, formar parte de una red de colaboradores que trabajan principalmente casos de niños y jóvenes objetos de diferentes injusticias. Esta cadena de apelantes, llamada Red Junior, permite pasar de la concienciación a la accción y en ella, colaboradores jóvenes, aun sin ser socios de Al, trabajan activamente por mejorar las condiciones de vida de otros jóvenes desfavorecidos o para denunciar aquellos gobiernos que incumplen su compromiso con la Declaración Universal de Derechos Humanos.

De este modo, las actividades de Al directa o indirectamente intentan contribuir a la educación sobre y en favor de los derechos humanos. En el curso de sus actividades diarias, los miembros de Al desarrollan lo mejor posible sus conocimientos y aptitudes al respecto. Sus actividades educan al mismo tiempo a las personas a quienes se trata de movilizar en la comunidad y a los sectores seleccionados como objeto de las acciones de Al, así como a los propios actores del trabajo en defensa de los derechos humanos.

\section{Otros aspectos prácticos de la educación de Derechos Humanos}

La educación de toda una comunidad en el tema de derechos humanos es sin duda un objetivo ambicioso, especialmente para Al u otras ONGs. A fin de potenciar sus resultados, las Secciones o estructuras estatales de AI han adoptado un gama de enfoques cada vez más concentrados y prácticos. Entre ellos figuran la captación de apoyos ante los gobiernos para intentar que se incluyan los derechos humanos en todos los niveles del programa de enseñanza y, en ocasiones, la prestación de asesoramiento al ministerio de educación o a las autoridades policiales en relación con ese programa. Algunas Secciones de AI han elaborado carpetas didácticas de EDH que sirven 
de base a esos programas de educación. También se realizan esporádicamente envíos de material educativo para tutorías a diferentes centros, se imparten ponencias en Cursos de Especialización del Profesorado y se oferta la colaboración en la ya citada Red Junior, bien por carta, bien mediante reuniones informativas, señalando también las posibilidades de las nuevas tecnologías al respecto.

Otras Secciones han concentrado la captación de apoyos en las ONG, ya que en ellas se encuentran personas altamente motivadas y capaces de difundir el contenido de la Declaración, creando así una suma de esfuerzos muy valiosa. En esa misma línea se realizan también charlas informativas a grupos juveniles en ámbitos extraescolares.

Por otro lado, en algunas sociedades hay también organismos nacionales de derechos humanos que cuentan con abundantes fondos e incluyen la $\mathrm{EDH}$ en su mandato. La captación de apoyo de estas organizaciones y de los gobiernos tiene como finalidad conseguir que una de sus tareas sea el fomento del conocimiento de los derechos humanos, y que se la tomen muy en serio, propiciando la publicación de material didáctico.

Las fuerzas armadas han sido otro de los objetivos de las gestiones nacionales dirigidas a que se organicen cursos de formación en derechos humanos para sectores específicos, como ya se ha señalado respecto a la formación de policías; ello ha obedecido en parte al incremento del número de gobiernos que aportan personal militar a las misiones internacionales de mantenimiento de la paz. Se ha sostenido que el papel y las competencias de los soldados que participan en estas misiones son bastante diferentes del correspondiente a los frentes de guerra convencionales, por lo que se trata de un ámbito todavía nuevo pero de creciente importancia. El número de misiones de este tipo va en aumento y, en algunos casos, participa en ellas personal de fuerzas militares que tienen un historial nacional de violaciones de derechos humanos importante. En consecuencia, Al procura tener acceso a los programas de formación de estos militares a fin de introducir el conocimiento de la Declaración Universal y constatar la obligación moral de dichas tropas de velar por su cumplimiento.

\section{Cómo integrar la educación y la acción}

Como ya hemos intentado explicar, $\mathrm{Al}$ tiene presente una serie de planteamientos prácticos en su trabajo intentando integrar lo mejor posible, la educación en derechos humanos con la acción en su defensa. Por eso, partiendo de que los miembros de los diferentes grupos locales participan en planes de formación periódicos a fin de mejor su conocimiento sobre los derechos humanos y los mecanismos dirigidos a protegerlos, puede entenderse que, posteriormente, se priorice su difusión mediante charlas, mesas redondas, etc, contribuyendo así a difundir la EDH, no sólo en el ambiente educativo, sino en el mundo adulto en general.

En el diseño de los diferentes planes de acción de AI, la EDH es un componente esencial por lo que se mantiene la adecuada coordinación entre los responsables de las diferentes Campañas y los responsables de EDH a fin de compartir material útil. Por ejemplo, un cartel creado para una campaña o un vídeo divulgativo sobre la situación de respeto a los derechos humanos en un determinado país, puede transformar- 
se en una herramienta de enseñanza si se envía con un folleto que proporcione sugerencias para su uso en el aula, de acuerdo con la edad idónea para ello.

En otras ocasiones, algunos sectores pertinentes de la sociedad son seleccionados como objetivo de campañas específicas dirigidas a la sensibilización de derechos humanos. A menudo es el mundo docente el receptor del material de estas campañas dada su especial formación e importancia, pero a veces, son otros sectores como el mundo judicial o el sindical.

Cuando procede, los llamamientos de denuncia a los gobiernos pueden incluir el ruego de que se introduzcan la educación sobre y en favor de los derechos humanos en sus programas educativos. Por ejemplo, en una campaña sobre un país donde los niños son víctimas de graves violaciones de derechos humanos, se puede pedir en los Ilamamientos a las autoridades, que las personas que trabajan directamente con niños reciban formación sobre los derechos de los niños y los mecanismos que los protegen.

Otra manera valiosa de educar es hacer participar a los jóvenes en acciones y campañas; esto puede hacerse oficialmente a través de contactos con centros de enseñanza y otros lugares, mediante la citada Red Junior u otro tipo de campañas como la llamada "Una postal, una vida", en la que los niños, niñas y jóvenes pueden colaborar activamente en la mejora de las condiciones de vida de otros jóvenes más desfavorecidos. También se propicia la realización de concursos de pintura y redacción sobre la Declaración Universal, la colaboración en actos conmemorativos del 10 de Diciembre, Día de celebración de la firma de dicha Declaración, del día 20 de Noviembre, Día internacional de los derechos del niño, o del Día de la Paz.

A modo de síntesis de lo ya descrito, aportamos los siguientes esquemas:

\section{OBJETIVOS DE UN PROGRAMA DE EDUCACIÓN EN DERECHOS HUMANOS}

Un programa de educación en derechos humanos tiene por objetivo permitir a los individuos adquirir conocimiento, comprensión y experiencia de:

- los conceptos de derechos humanos y los valores y actitudes fundamentales que conducen al respeto por estos derechos;

- los instrumentos que recogen y protegen los derechos humanos

- las habilidades, valores y actitudes que defienden la igualdad de derechos para todos y mueven a la acción para proteger estos derechos.

OBJETIVOS DE UN PROGRAMA DE SENSIBILIZACION SOBRE LOS DERECHOS HUMANOS

Un programa de sensibilización sobre los Derechos Humanos tiene por objetivo ayudar a que los individuos reconozcan la existencia, pertinencia e importancia de:

- los instrumentos que recogen y protegen los derechos humanos

- la necesidad de promover y proteger los derechos humanos

- la labor de derechos humanos que realizan las organizaciones gubernamentales y no gubernamentales 


\section{ELEMENTOS DEL PROGRAMA DE EDUCACION EN DERECHOS HUMANOS DE AI}

El programa de EDH de Al incluye:

- la captación de apoyos para lograr la incorporación de la EDH a los programas oficiales de formación y educación en centros de enseñanza, academias militares y de policía y cursos de administración pública;

- la participación en programas de formación y educación ya sea que los organice Al, otras organizaciones o entes oficiales, aportando la creación de material didáctico específico, apoyando el trabajo del profesorado especialmente en ética y tutorías.

- la organización, sola o con otras organizaciones, de actividades de educación informales, como espectáculos callejeros, teatros de marionetas para niños, concursos literarios y artísticos para jóvenes, películas y programas de radio y televisión, que difundan el contenido de la Declaración Universal de Derechos Humanos;

- apoyar y facilitar el trabajo de los educadores en derechos humanos dentro y fuera de $\mathrm{Al}$, principalmente mediante la participación en redes regionales y nacionales de educación en derechos humanos.

\section{Material didáctico de apoyo}

Los libros y manuales editados por Al pretenden ser un instrumento auxiliar para el trabajo de maestros, profesores y demás personas encargadas de tareas docentes en diversos ámbitos: escuelas, institutos, colegios, etc. Se proporciona material específicamente dirigido a los profesores y a los alumnos, así como material de uso indistinto. Se plantea una clara separación de temas que permita trabajar de modo exclusivo aquellos que se consideren más oportunos, bien por razón del tiempo disponible, bien por la edad o tipo de alumnos con los que se trabaje. Se plantean como objetivos generales el conocimiento de los derechos humanos reconocidos en la Declaración Universal de la ONU y de las violaciones de los mismos a nivel mundial, la sensibilización de las personas sobre la importancia social y personal del reconocimiento y el respeto de estos derechos, así como el fomento de una postura activa en defensa de los derechos humanos, tanto en alumnos como en profesores.

El material elaborado tiene, en consecuencia, carácter abierto y se parte de un enfoque socioafectivo que permita tratar aspectos relacionados con la paz y el desarrollo en los diversos niveles educativos. Se constata que aún escasea el material adecuado para tratar estos temas más allá de la explicación de grandes textos y declaraciones y por eso, el material desarrollado se concibe como algo que no sólo trasciende las meras palabras sino que es inseparable del propio ambiente educativo, partiendo de la convicción de que no se educa con palabras sino, sobre todo, con actos.

Un ejemplo de lo dicho hasta aquí lo constituye el texto "Educación en Derechos Humanos. Propuestas didácticas." de la editorial "Los libros de la catarata" en 1.995. En el se desarrollan diferentes bloques temáticos proporcionando dinámicas de grupo, textos de apoyo, testimonios, como se apuntan brevemente a continuación: 
LOS PRESOS DE CONCIENCIA: Ejemplos históricos (Sócrates, Jesús de Galilea, Galileo, Gandhi...). Situaciones reales en Grecia, Colombia, Cuba, etc. Se apuntan ejemplos de cartas y telegramas para trabajar activamente por la liberación de los presos de conciencia.

LA PENA DE MUERTE: Consideraciones generales, formación de opinión por parte del alumnado reflexionando argumentos a favor y en contra. Se parte de datos y estadísticas reales, proporcionando dramatizaciones, encuestas, textos de reflexión, etc.

CASOS DE ADOPCION DE AMNISTIA INTERNACIONAL: Relación de casos reales. Dinámicas de contextualización.

LA TORTURA: Cuestionarios, técnicas de investigación, reflexión sobre el Programa de doce puntos para la abolición de la tortura realizado por Amnistía Internacional. Síntesis histórica.

JUICIOS IMPARCIALES Y EXPEDITOS: Reflexión a partir de las películas "El proceso", "Falso culpable" y "Doce hombres sin piedad". Dinámicas de grupo. Dramatización de un juicio real.

HISTORIAS PARA TRABAJAR LOS DERECHOS HUMANOS: Su finalidad es analizar alternativas de solución ante hechos antisociales o delictivos, distinguir entre justicia y castigo y comprender la irracionalidad de la Ley del Talión, tomando postura contra la pena de muerte.

INFORMACION COMPLEMENTARIA: Se aportan testimonios reales de "buenas noticias", casos en los que el trabajo de AI ha conseguido la liberación de presos de conciencia, así como el texto completo de la Declaración Universal de Derechos Humanos. En cuanto a bibliografía se aportan ensayos, títulos de literatura tanto para adultos como infantil y juvenil, y una relación bastante exhaustiva de películas interesantes en derechos humanos.

Otra vía importante de trabajo en educación por los derechos humanos son los talleres de formación realizados por educadores especialmente sensibilizados que proporcionan a otros compañeros importante información y dinámicas de trabajo en el aula, especialmente diseñadas para tutoría.Un ejemplo muy valioso es la publicación fruto del seminario de Educación por la paz llevado a cabo por la Asociación Pro Derechos Humanos. El resultado es el libro titulado "Educar en y para los Derechos Humanos. Dinámicas y actividades." editado por "Los libros de la catarata" en 1.996.

La filosofía del texto parte de la base de que sería difícil encontrar al menos en los países occidentales, a alguien que negase la importancia de conocer y divulgar en el marco escolar la Declaración Universal de los Derechos Humanos. Además existen numerosas declaraciones, recomendaciones y directrices procedentes de diversos organismos internacionales que proponen objetivos, pautas, metas y que, a veces, relacionan la educación sobre derechos humanos con la educación para el desarrollo, la educación para la cooperación y cooperación internacional, la educación para la paz, etcétera. Existen incluso excelentes proyectos curriculares con buenos materiales de apoyo. 
Sin embargo, la tarea de sensibilización educativa falla con frecuencia a la hora de transmitir a los adolescentes una imagen positiva de la constante tensión entre el interés por los derechos humanos y la constante violación de éstos en todo el planeta. Resulta descorazonador oir con cierta frecuencia que los textos al respecto son papel mojado. Nosotros consideramos que es preciso transmitir en las aulas que los derechos contenidos en la Declaración Universal son una conquista de la humanidad que exige una lucha permanente para darle vigencia, completarlos, ejercerlos, algo que, en suma, nos obliga a ser constantemente responsables. No basta con que estén declarados y escritos; tenemos que que hacerlos realidad en nosotros mismos y en nuestros pueblos. Y, más aún, hemos de empezar por la propia comunidad escolar: el curriculum oculto, lo que enseñamos en el ambiente, las relaciones sociales y las actitudes cotidianas en los centros pueden conseguir que se desaprenda buena parte de lo que afirmamos explícitamente en nuestras clases.

Las propuestas didácticas del texto se han pensado para combinar la educación sobre derechos humanos, la educación en derechos humanos y la educación para los derechos humanos. Así pues, se presentan pautas, herramientas de trabajo y sugerencias de actividades que difícilmente servirán para algo si se aplican dentro de lo que Paulo Freire definía como una concepción bancaria, meramente pasiva y transmisiva de la educación. Educar para los derechos humanos es difícil: evidentemente, es necesario informar, conocer el articulado de la declaración, saber que su contenido no se limita al simple enunciado de los 30 artículos.

El abismo entre las palabras, el discurso en el aula, y los hechos y las actitudes es el primer obstáculo a vencer para educar en y para los derechos humanos. No se puede aprender algo que no se practica. Este obstáculo se puede salvar con dos acciones combinadas:

a) a través del Ilamado enfoque socio-afectivo, es decir, concibiendo el proceso de información sobre los derechos humanos como un aprendizaje en la propia piel, partiendo de situaciones educativas que implican experiencias vivenciales, juegos de roles y de simulación, dramatizaciones... que nos permiten hacer aflorar sentimientos y actitudes profundas.

b) situando la reflexión y la práctica de los derechos humanos al alcance de la experiencia inmediata de los chicos y chicas, es decir, enmarcándola en la propia actividad educativa y transmitiendo un compromiso serio de hacerlos realidad en la propia escuela..., a la vez que se les ayuda a descubrir que tienen unos derechos inalienables. Es lo que podríamos Ilamar la contextualización de los derechos humanos en la propia escuela.

A lo largo de estos últimos años, la importancia de los problemas de especie que afectan a la humanidad ha hecho que apareciesen tareas educativas parciales. Así, se habla de la educación para la paz, educación ambiental, educación para el desarrollo y, naturalmente, educación para los derechos humanos. Podemos entender cada una de estas cuatro educaciones en un sentido restringido o en un sentido ampliado. Como se puede ver en el caso de la educación para derechos humanos, la concepción ampliada presupone que estos cuatro conceptos son complementarios, interdependientes y mutuamente reveladores los unos de los otros. 
EDUCACION PARA LOS DERECHOS HUMANOS (ENFOQUE RESTRINGIDO)

a) Actividades educativas basadas en los documentos internacionales clave.

b) Enfasis en los derechos civiles y políticos.

c) Aceptación implícita de una visión con frecuencia occidentalizada de los derechos.

d) Enseñanza sobre los derechos ( su historia, estudio de casos específicos...).

EDUCACION PARA LOS DERECHOS HUMANOS (ENFOQUE AMPLIADO)

a) Consideración de los nuevos derechos, es decir, de los derechos ambientales desde una perspectiva que contempla también los derechos de las generaciones futuras a disfutar del planeta y de sus recursos, incluyendo los recursos no renovables.

b) Enfasis por igual en derechos sociales y económicos y en los derechos civiles y políticos.

c) Tener seriamente en cuanta las perspectivas no occidentalizantes a la hora de considerar los derechos

d) Enseñanza para los derechos humanos (es decir, enseñanza no sólo de contenidos sino también de hábitos, de recursos que permitan el ejercicio y la defensa de estos derechos) y en un ambiente respetuoso de estos derechos (es decir, consideración de las actividades del grupo clase y de la escuela como de un lugar donde se deben respetar y ejer cer, con las obvias diferencias de los niveles de edad, estos derechos).

A partir de estas orientaciones pedagógicas previas y una vez matizados los dos enfoques de enseñanza diferentes citados, se desarrolla el texto, de acuerdo con el siguiente sumario que, por su interés, transcribimos a continuación.

Cap. 1: Actividades relacionadas con los conceptos básicos de los derechos humanos Introducción

¿Qué son los derechos humanos?

Un nuevo planeta, ¿unos nuevos derechos?

Los derechos agrupados por categorías

Los derechos humanos y el lenguaje

Los derechos humanos a través de la expresión artística

Los derechos humanos básicos y su violación

El futuro de los derechos humanos

La expresión de los propios valores

Cap.2: Actividades relacionadas con el estudio de algunos derechos humanos en concreto

1. Art.1: derecho a la libertad e igualdad

1.1. Comentario del texto del artículo

1.2. Una escala de valores: egocentrismo y fraternidad

1.3. Situaciones vivenciales

1.4. Lectura y comentario de El Señor de las moscas 
2. Art.3: derecho a la vida, la libertad y la seguridad

2.1. Comentario del texto del artículo

2.2. Comentario de textos literarios

2.3. Juego de simulación: las relaciones entre libertad, paz y justicia

3. Art.5: derecho a la integridad personal

3.1. Comentario del texto del artículo

3.2. Comentario de la Convención Internacional contra la Tortura

3.3. Comentario de textos literarios

3.4. Torturas y malos tratos a niños

4. Arts. 10 y 11: derecho a la presunción de inocencia y a un juicio justo

4.1. Comentario del texto de los artículos

4.2. Análisis de un texto relativo a la reforma de la Administración. de justicia en España

4.3. Cómo cambiar de punto de vista: un lobo no tan feroz

4.4. Búsqueda de información: ONGs que trabajan contra el racismo y la xenofobia

5. Art.15: derecho a la nacionalidad

5.1. Comentario del texto del artículo

5.2. Comentario de textos literarios

5.3. Dinámica: Ser un extraño

6. Art.18: derecho a la libertad de creencias

6.1. Comentario del texto del artículo

6.2. Análisis de textos literarios

6.3. Situaciones vivenciales

7.Art.25: derecho a un nivel de vida adecuado

7.1. Comentario del texto el artículo

7.2. Trabajo a partir de vídeos: Hambre y alimentación

7.3. Hambrunas que no caen del cielo

8. Art.26: derecho a la educación

8.1. Comentario del texto del artículo

8.2. Un derecho relacionado con otros derechos

8.3. Investigación sobre el estado de la educación en el mundo

8.4. Estudio de casos: discriminación por SIDA

Cap.3: Situaciones y formas específicas en las que se violan los derechos humanos

1. Diversos tipos de discriminaciones

1.1. Mundo de colores

1.2. La cinta amarilla

1.3. La experiencia en el Instituto Eugeni d,Ors de Badalona

1.4. Vídeo: La clase dividida 
2. Los refugiados

2.1. ¿Qué es el ACNUR?

2.2. Análisis de un testimonio personal

2.3. Dinámica: la familia de Hamdi

3. Los derechos de lasa niñas y los niños

3.1. Lectura y comentario de la Declaración de los derechos del niño

3.2. Ejemplos de actuaciones correctoras: Siendo, de Eduardo Galeano

4. Los derechos de los pueblos

4.1. Dinámica: la cultura Nacirema

4.2. Nuevo Orden Económico Internacional: historia de Tritonia y Nortonia

4.3. Deuda externa y carrera de armamentos

4.4. Deuda externa: actividad final

Cap. 4: Organizaciones y procedimientos para proteger los derechos humanos

1. Simulación de un encuentro de la Comisión de Naciones Unidas sobre Derechos Humanos

2. Un juego de roles sobre denuncias de violaciones de derechos humanos en la UNESCO

3. El papel de las ONGS en los derechos humanos: Amnistía Internacional y Madres de la Plaza de Mayo

4. 50 años de Naciones Unidas

5. Organos de Naciones Unidas

6. Negociar para el futuro: reforma de las Naciones Unidas

Cap. 5: Ampliando la Declaración Universal

1.Una cuestión polémica

1.1. Un debate sobre la violencia de ETA (X. Etxebarría)

1.2. Alternativas para la Paz: Elkarri y Coordinadora Gesto por la Paz

2. Los derechos de la tierra

2.1. Lectura, comentario y dramatización de la carta del indio Seattle

2.2. Un nuevo apartado de la Declaración: los derechos de la tierra

2.3. Escribamos nuestra propia declaración

Cap. 6: Los derechos humanos en nuestros centros educativos

Derechos humanos en el centro educativo

1. Una Carta de Derechos y Responsabilidades

2. Métodos y estructuras de consulta y participación

3. Procedimientos y mecanismos para resolver quejas, agravios, conflictos,etc.

Una propuesta final: El Gran Dictador

Libros y recursos: Bibliografía empleada, Materiales sobre derechos humanos y

Grupos de derechos humanos y educación por la paz 


\section{Bibliografía}

Amnistía Internacional. 1996. Educar en y para los Derechos Humanos. Propuestas didácticas. Madrid: Los libros de la catarata.

Amnistía Internacional. 1995. Educación en Derechos Humanos. Propuestas didácticas. Madrid: Los libros de la catarata.

Amnistía Internacional. 1980. Declaración Universal de Derechos Humanos. Madrid.

BARREIRO, C. 1981. Derechos Humanos. Declaraciones solemnes, continuas violaciones. Barcelona: Salvat.

LEAH LEVIN. 1981. Los Derechos Humanos: Preguntas y respuestas. París: UNESCO. MANOS UNIDAS. 1985. Hagamos un solo mundo. Madrid: IEPALA.

Instituto Nacional del Libro Español. Libros para la paz. Madrid: Ministerio de Cultura.

TUVILLA RAYO, J. 1998. Educación en derechos humanos: hacia una perspectiva global. Bilbao: Desclée de Brouwer.

GARCÍA MORIYÓN, F. 1999. Derechos humanos y educación: textos fundamentales, textos complementarios. Madrid: Ediciones de la Torre.

JARES, X. R. 1999. Educación y derechos humanos: estrategias didácticas y organizativas. Madrid: Editorial Popular.

AGUARELES, M. A. y otros. 1991. La educación moral. Perspectivas de futuro y técnicas de trabajo. Barcelona: Institut de Ciències dell Educació, Universitat de Barcelona. 